\title{
Force and thickness prediction with FEA of the cranial implants manufactured through SPIF
}

\author{
Mihai Popp ${ }^{1}$, Gabriela Rusu ${ }^{1}$, Sever-Gabriel Racz ${ }^{1}$, and Ilie Octavian Popp ${ }^{1}$ \\ ${ }^{1}$ University "Lucian Blaga" of Sibiu, Department of Industrial Machinery and Equipment, Emil \\ Cioran 4, Sibiu, Romania
}

\begin{abstract}
Single point incremental forming is a process which is primarily used for prototyping, small series production and unique parts. This procedure shows a high potential in the development of complex shape parts where the time of the execution does not play an important role. Among the applications of this process are the execution of various medical prostheses, such as: knee prostheses and cranial implants. The aim of this paper is to determine the forces which take place during the manufacture process of an cranial implant, as well as the thinning of the material through the help of finite element analysis. After the numerical simulation, from the results obtained it is possible to observe the behaviour of the material at different forces which appear during the deformation process. In addition, in this paper will be presented considerations about the thinning of the material used for the cranial implant.
\end{abstract}

\section{Introduction}

Today's medicine has reached such an advanced level that it allows the replacement of parts of the body with artificial ones known as prostheses. The most common artificial prostheses or implants are those that replace the arms, the legs, teeth and eyes. These surgical reconstructions of parts of the body, damaged by illness or trauma, restore the patient's lifestyle. There are situations where implants are needed to save a person's life, such as the cranial implant which is currently attracting more and more attention.

The skull or cranium is a critical part of the human body, being the bone structure which contains the brain and has the role to protect it. As a result of contusive lesions, cranial bleeding, or the removal of a brain tumour, there may be a need to remove a bone fracture from the skull. If it is not possible to restore the integrity of the patient's skull with his own bone, there is a need to replace the missing part with pieces made out of metal or resin plates or structures that exhibit mechanical properties similar to human bone. The procedure for repairing a defect or malformation in the skull is called cranioplasty.

Such a cranial implant has the particularity that it must be customized according to the constitution of each patient. Since 1998 due to the rapid evolution of medical imaging and 3D modelling, custom implants using computer-aided design and manufacturing programs (CAD and CAM) have been achieved [1]. The steps that should be followed in order to achieve cranial implants are presented in Figure 1. 


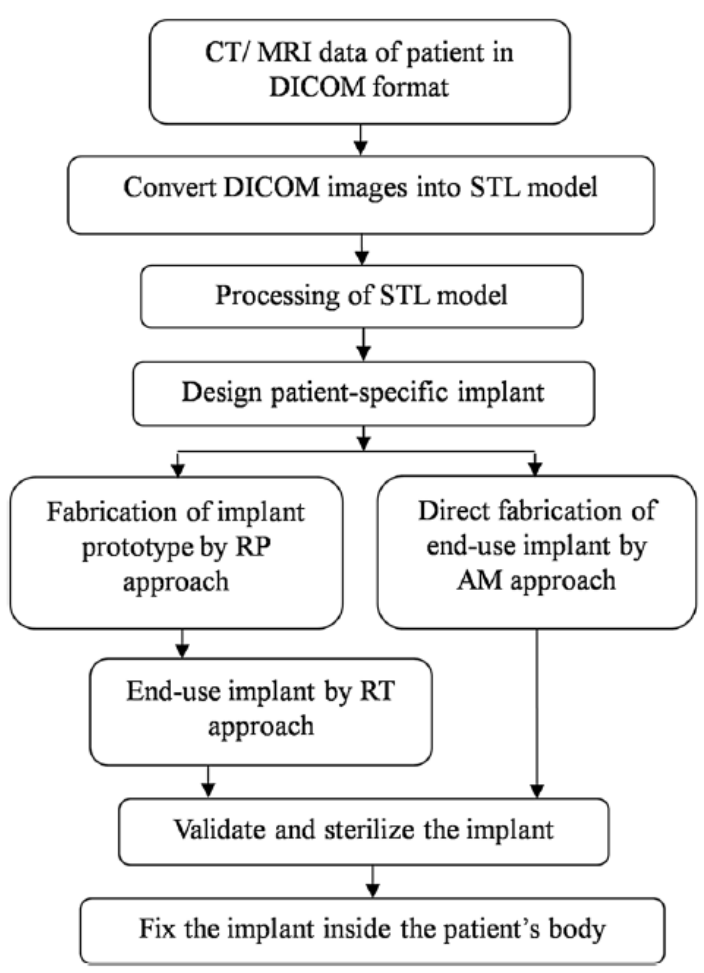

Fig. 1. Steps to fabricate patient-specific implant [2]

Due to the high degree of customization for each implant, the use of processes favouring the production of unique parts is suitable for their manufacture. One of the manufacturing processes that is suitable for producing the cranial implants is incremental deformation. It is a technology that is widely used in the field of automotive and aeronautic industries. This way of manufacturing the cranial implants brings a great benefit also in the medical industry because the implant-processing technicians do not have to deal with many attempts until they reach the desired shape and size, therefore it can be done in a single attempt. At the same time, in order to reduce time and cost, it is a good practice to use the finite element analysis method that provides information so that the achieved part is the optimal one.

In the article [3] it is proposed to implement a specific platform to produce biomedical complex components. The studies were carried out on knee prosthesis and a number of parameters were discussed. From this parameters the focus was on how to improve the surface quality and roughness. The studies concluded that the height of the step on $\mathrm{Z}$ axis has a big influence on the quality of the component and the roughness is dependent with the spindle speed.

For the manufacturing of cranial implants, a variety of resemblance materials as resistance properties to human bone have been used, such as: various resins, acrylic cements or various metals (steels or titanium). The present technology allows the development of cranial implants by incremental deformation made of metals.

The incremental deformation process enjoys an increasing popularity among researchers around the world due to the high degree of freedom it poses to the complexity of the parts geometry. The principle of the incremental sheet forming process consists in the use of a punch that moves on a specific trajectory applying pressure on the semi finished. The trajectories followed by the punch can have a high degree of complexity. In the next Figure it is possible to observe the semi finished 2 which is fastened between the active plate 4 and 
the retaining plate 3. During the incremental sheet forming of the semi finished, this remains fixed and the punch deforms the plastic sheet, thus reaching the finished part.

The incremental sheet forming process can be accomplished with the help of CNC machines or industrial robots. Currently there is still no dedicated software packages to generate the trajectories for the single point incremental forming process, so the researchers can use the common CAM software designed for milling machines. [4]

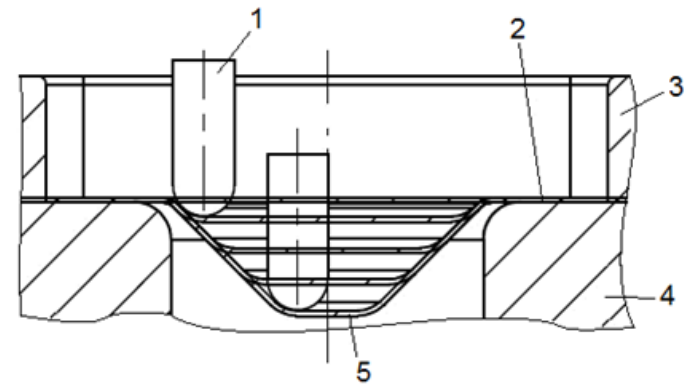

Fig. 2. Single point incremental forming process principle [4]

In the paper [5] several test were conducted on different plates mounted on polymethylmethacrylate supports for the cranial implants. Regarding the material used for the plates, there were used three different types of titanium: pure titanium and two alloys of Ti-6AL-4v. After the plates were mounted on supports, there were carried out several drop tests on flat surfaces in order to verify the mechanical strength of cranial implants. The results show no influence of the procedure of manufacture for the cranial implants and no failure of the prosthesis in both areas: impact zone and anchoring region.

\section{Force and thickness prediction with finite element analysis}

This paper presents the method to determine the forces emerged during the manufacturing of a cranial implant through single point incremental forming process and the thinning of the material used, in order to optimize the physical process. The prediction of forces and material thinning can be done with the help of different finite element analysis software packages. Knowing the values of the maximum forces that can occur during this process help researchers to prevent material degradation and at the same time to choose the suitable equipment that can perform the incremental deformation process on the material used. Furthermore, according to a study done in the paper [6], force prediction helps correcting the trajectories made by the punch so that the final shape and precision of the desired piece will be improved, which is extremely important for the cranial implants.

Starting from the 3D model of a skull, designed in the CATIA software shown in Figure 3 , a part of it was extracted, which will be considered the desired shape of the cranial implant for further investigations regarding the aim of this paper. 


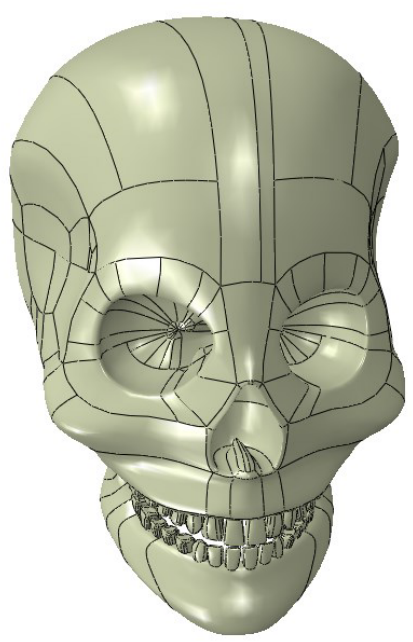

Fig. 3. 3D representation of the skull
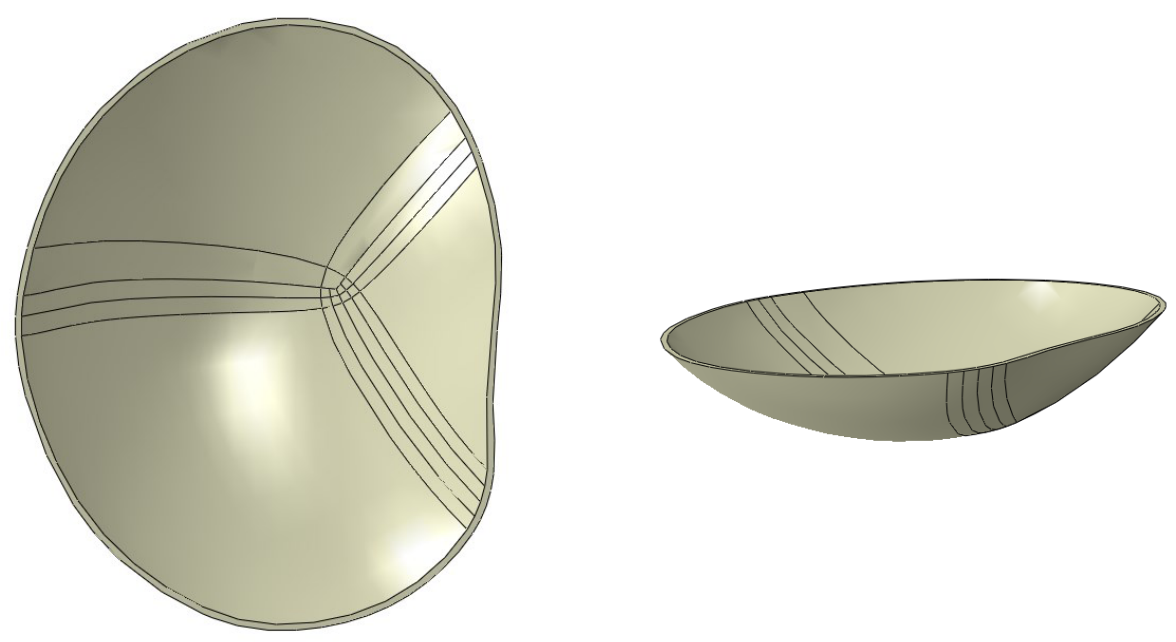

Fig. 4. 3D representation of the chosen cranial implant

In order to achieve the goal of the paper, a finite element analysis was carried out with the chosen implant. In the first step the cranial implant was discretized using a specific orientation of the elements, in such a way to obtain a spiral path on the cranium. Afterwards the $\mathrm{X}, \mathrm{Y}$ and $\mathrm{Z}$ coordinates of the nodes on this path were manually extracted and introduced in ABAQUS software. In the end, after combining all the points we obtain the spiral path which the tool needs to follow for this customized cranial implant. This trajectory is shown in Figure 5. 


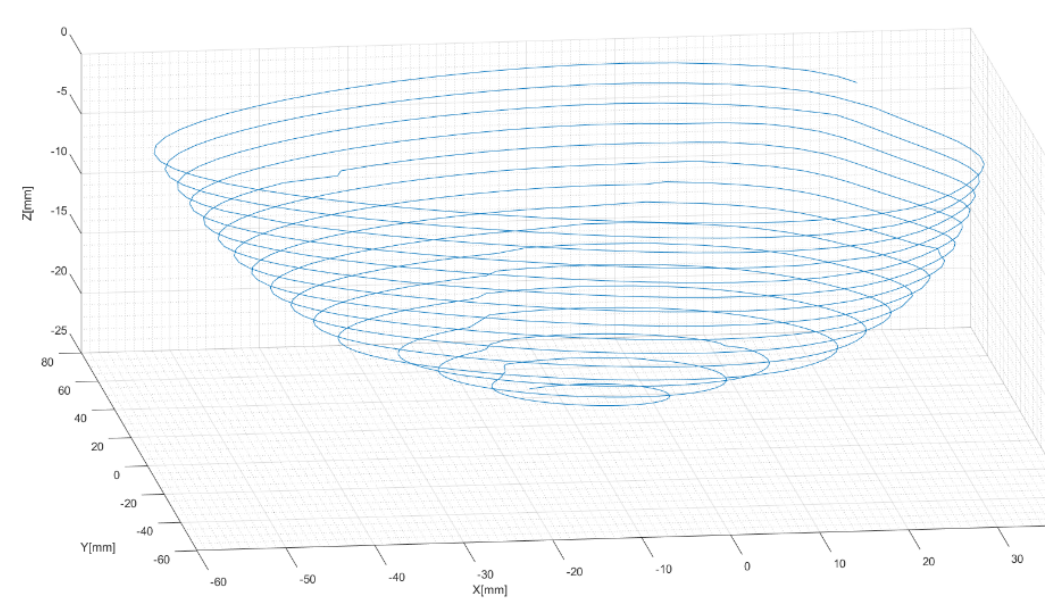

Fig. 5. Trajectory used for manufacturing the cranial implant

The numerical simulation is performed using ABAQUS / Explicit finite element analysis program. The model used consists of a rigid analytical retaining ring, a deformable sheet metal made of steel and a rigid analytical tool. The thickness of the sheet for this model is $4 \mathrm{~mm}$. The steel sheet is meshed with 4-node, quadrilateral, stress / displacement shell elements with reduced integration and a large-strain formulation (S4R). The material used as a semi-finished product is S700, which is a steel alloy and has the following chemical concentration: $\mathrm{C}-0,12 \%, \mathrm{Si}-0,21 \%, \mathrm{Mn}-2,1 \%, \mathrm{P}-0,02 \%, \mathrm{Al}-0,015 \%, \mathrm{Nb}-0,09 \%, \mathrm{~V}$ $-0,2 \%$ and $\mathrm{Ti}-0,15 \%$. This material has the following properties: Yield strength is 700 $\mathrm{MPa}$, tensile strength is $750-950 \mathrm{MPa}$, percentage of elongation is $10 \%$. In ABAQUS was introduced the plasticity of the material which was determined with the help of von Mises flow criterion.

The parameters targeted after following all the steps necessary to prepare the preprocessing and after solving the analysis: major strains, minor strains, material thinning, von Mises stress and the displacement on $\mathrm{Z}$ axis. Figures 6 and 7 presents the variations in 1 and 2 for the cranial implant that was simulated for this research and can be observed that the maximum value for 1 is $36,6 \%$, respectively $13,9 \%$ for the 2 .

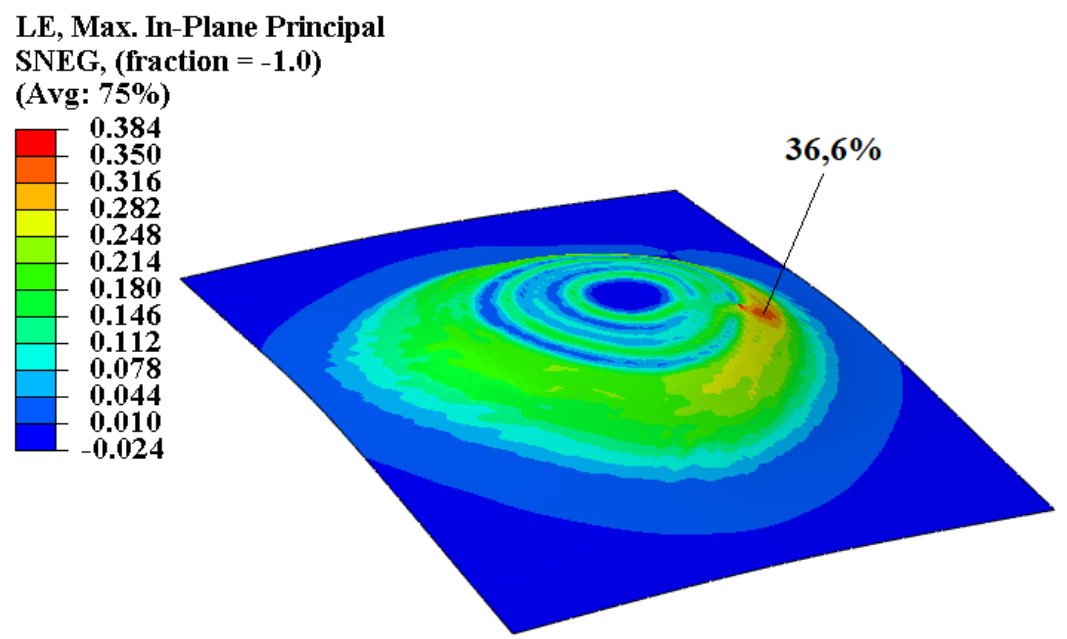

Fig. 6. Distribution of major strains $\left(\begin{array}{l}1 \\ 1\end{array}\right)$ on the cranial implant simulated 


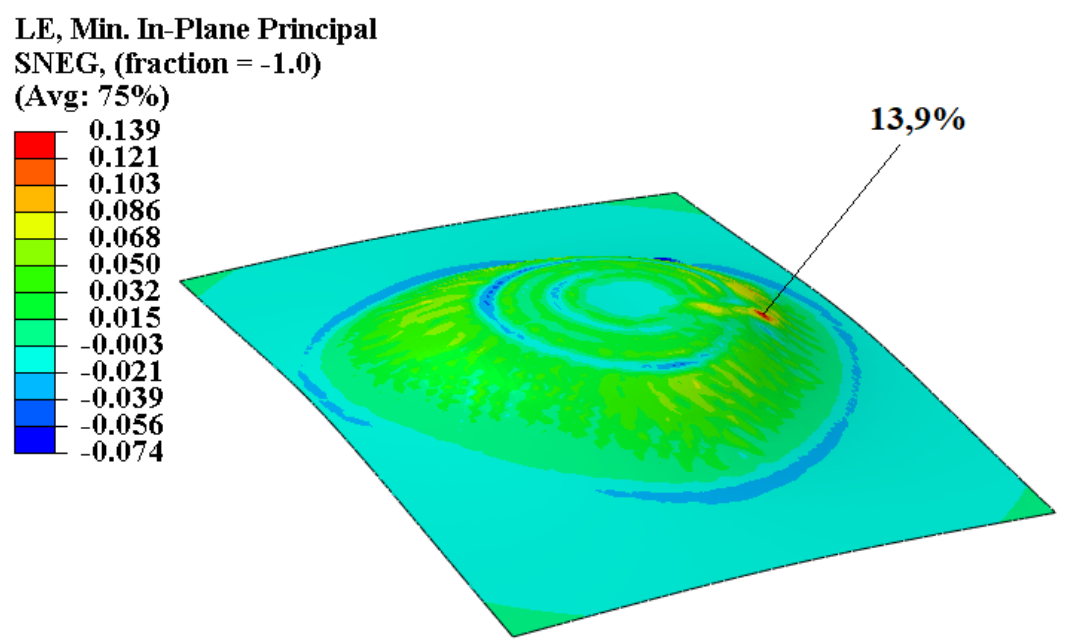

Fig. 7. Trajectory used Distribution of minor strains $(2)$ for the cranial implant simulated

Von Mises stress reveals that the maximum stress value of $627 \mathrm{MPa}$ located on the top of the sheet metal does not exceed the acceptable limit of yield strength. This result is presented in Figure 8 and shows that the material is still in the elastic domain deformation.

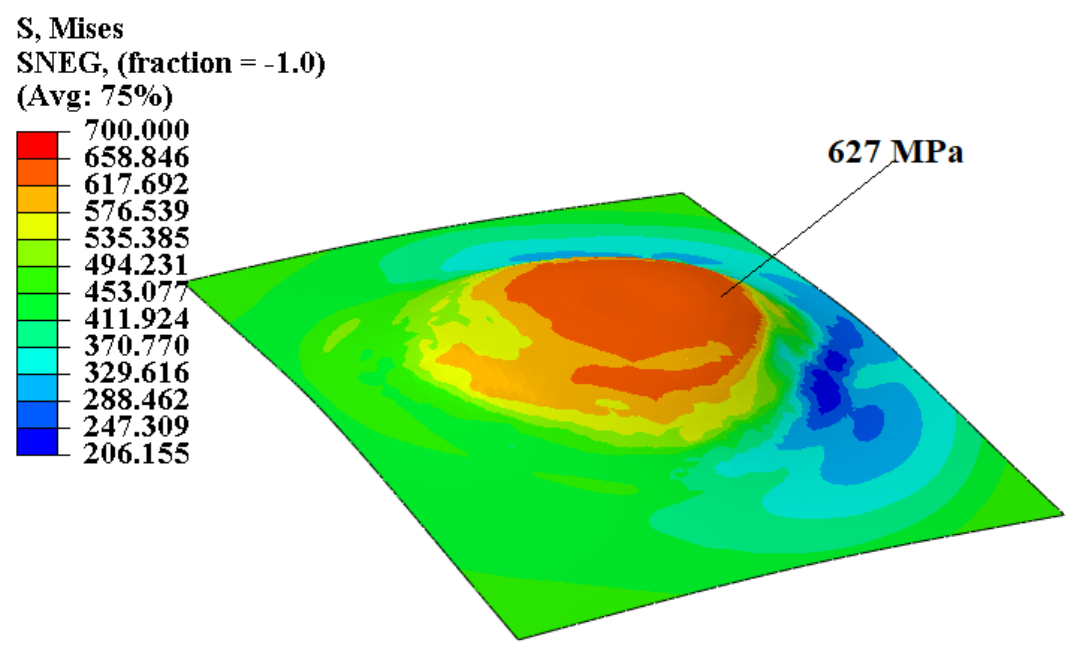

Fig. 8. Distribution of von Mises stress on the cranial implant simulated

On the Figure 9 is presented the distribution of thickness reduction on the sheet metal used for the cranial implant and the maximum value is $46 \%$. 
STH

(Avg: 75\%)
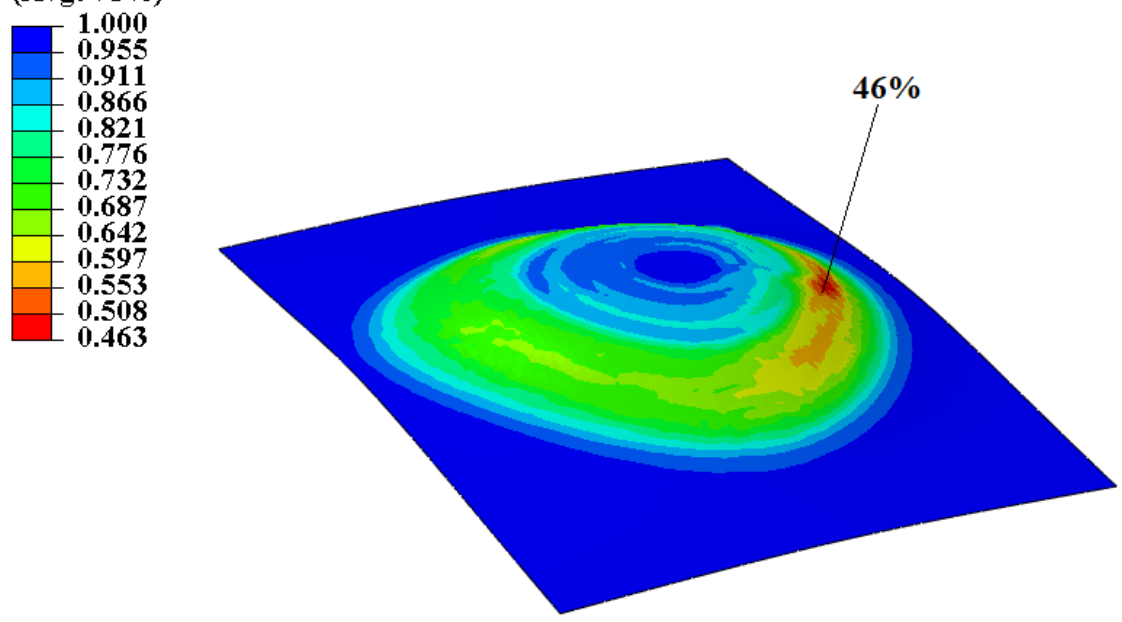

Fig. 9. Distribution of thickness reduction on the cranial implant

From Figure 10 it can be observed that the material is in the elastic domain from the Force - Displacement diagram. The maximum value of the predicted force can be observed in the diagram presented below and has the value of $25,9 \mathrm{kN}$.

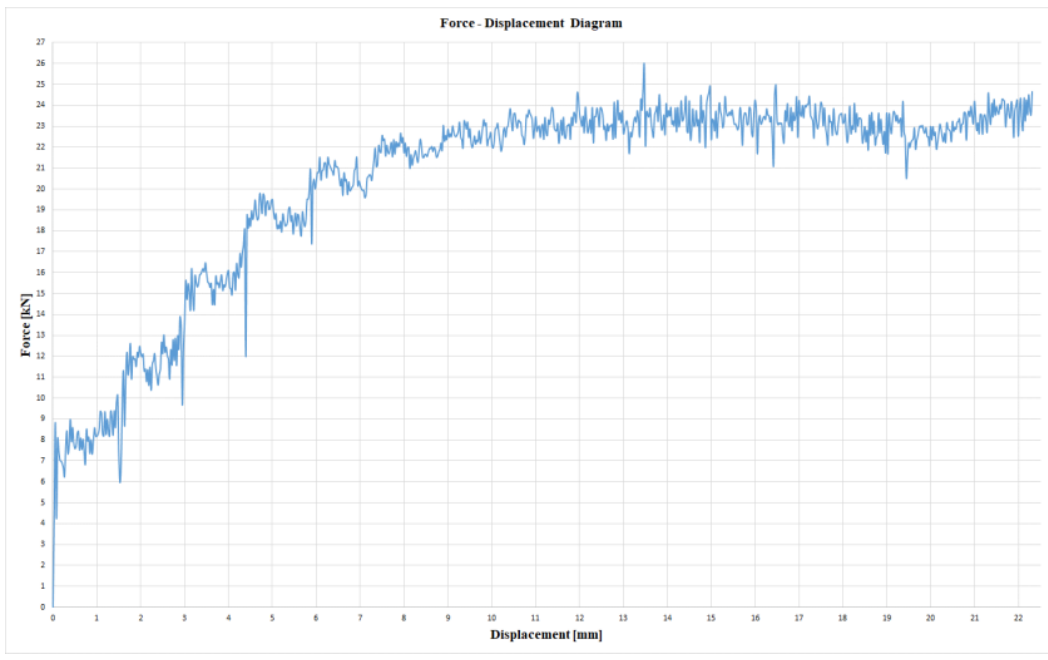

Fig. 10. Force - Displacement Diagram

\section{Conclusion}

It's a good practice to use finite element method analysis to predict the force and thickness reduction because the incremental sheet forming process has a high degree of complexity. Knowing the force before doing the procedure, it can prevent the failure of the material and helps to correct the trajectory of the tool in order to obtain more precise geometries of parts and more appropriate to the desired shape. It's important to know the thickness reduction before doing the procedure from the simulation because it is possible to change the material 
of the part in such a manner to obtain a part that withstand to the process. The use of explicit dynamic analysis for the studied process has the advantage that it is possible to determine the moment when material failure appears.

From the simulation performed it can be observed that a sheet metal made of S700 with the thickness of $4 \mathrm{~mm}$ can resist to the single point incremental forming process. This can be observed in Figure 8 where the maximum stress value is $627 \mathrm{MPa}$ and does not exceed the acceptable limit of yield strength and also from the Figure 9 where the maximum thickness reduction is $46 \%$. The maximum predicted force can be observed in the Force - Displacement Diagram and has the value of $25,9 \mathrm{kN}$.

In further research, we aim to perform various experiments to validate the presented results obtained from the numerical simulation performed on the chosen cranial implant.

\section{References}

1. https://www.academia.edu/7611433/Cranioplastie

2. Y. K. Modi, S. Sanadya, Journal of the Brazilian Society of Mechanical Sciences and Engineering, Brazilia, (2018)

3. A. Boulila, M. Ayadi, S. Marzouki, S. Bouzidi, The International Journal of Advanced Manufacturing Technology, London, (2018)

4. J. Jeswiet, F. Micari, G. Hirt, A. Bramley, J. Duflou, J. Allwood, Annals of CIRP, (2005)

5. G. Ambrogio, G. Palumbo, E. Sgambitterra, P. Gulielmi, L. De Napoli, T. Villa, G. Fragomeni, The International Journal of Advanced Manufacturing Technology, (2018)

6. J. Belchior, D. Guines, L. Leotoing, E. Ragneau, Key Enginnering Materials, (2013)

7. R. Aerens, P. Eyckens, A. Van Bael, J.R. Duflou, Internationl Journal Advance Manufactured Technology, (2010)

8. D. Bhargava, P. Bartlett, J. Russell, M. Liddington, A. Tyagi, P. Chumas, Acta Neurochir, (2010)

9. G. Centeno, I. Bagydanch, D. Morales-Palma, M.L. Garcia-Romeu, B. Gonzalez-PerezSomarriba, A.J. Martinez-Donaire, L.M. Gonzalez-Perez, C. Vallellano, $17^{\text {th }}$ International Conference on Sheet Metal, (2017)

10. J. R. Duflou, A.M. Habraken, J. Cao, R. Malhotra, M. Bambach, D. Adams, H. Vanhove, A. Mohammadi, J. Jeswiet, International Journal of Material Forming, France, (2018)

11. M. Crenganiș, ICAMaT, Romania, (2018)

12. V. Oleksik, Procedia Engineering, (2014)

13. A. Blaga, V. Oleksik, Advances in Materials Science and Engineering, (2013) 